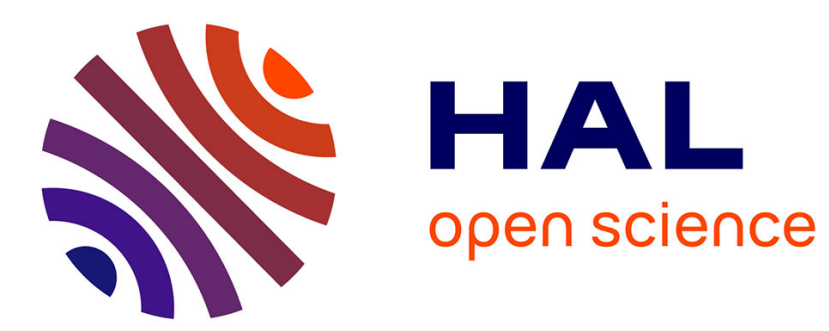

\title{
Open shell organometallics: a general analysis of their electronic structure and reactivity \\ Rinaldo Poli
}

\section{To cite this version:}

Rinaldo Poli. Open shell organometallics: a general analysis of their electronic structure and reactivity. Journal of Organometallic Chemistry, 2004, 689 (24), pp.4291-4304. 10.1016/j.jorganchem.2004.05.040 . hal-03278363

\section{HAL Id: hal-03278363 https://hal.science/hal-03278363}

Submitted on 6 Jul 2021

HAL is a multi-disciplinary open access archive for the deposit and dissemination of scientific research documents, whether they are published or not. The documents may come from teaching and research institutions in France or abroad, or from public or private research centers.
L'archive ouverte pluridisciplinaire HAL, est destinée au dépôt et à la diffusion de documents scientifiques de niveau recherche, publiés ou non, émanant des établissements d'enseignement et de recherche français ou étrangers, des laboratoires publics ou privés. 
Open Shell Organometallics: a General Analysis of their Electronic Structure and Reactivity

\author{
Rinaldo Poli
}

Laboratoire de Chimie de Coordination, 205 Route de Narbonne, 31077 Toulouse Cedex,

France

Proofs to: Rinaldo Poli

$+33-561333195$

fax $+33-561553003$

poli@lcc-toulouse.fr

http://www.lcc-toulouse.fr/equipes/equipe_g/pages personnelles/poli/index.html 


\begin{abstract}
Transition metal organometallic compounds that contain fewer than 18-electrons and two or more unpaired electrons are generally excluded from treatises of either Werner-type coordination compounds or organometallic chemistry. However, they can be seen as the bridge filling the gap between these two traditional areas of coordination chemistry. Their magnetic and optical properties are reminiscent of the Werner-type complexes, whereas their chemical reactivity parallels that of the lower-valent organometallics. Spin state change phenomena are of paramount importance in this area. This article provides a broad perspective of this area, with particular attention to: $(i)$ how the ground state properties can be related to the metal and ligands nature; (ii) under which circumstances the often inappropriately invoked concept of "spin block" is meaningful; (iii) the spin acceleration concept; (iv) how the coordination sphere affects the topology of the reaction coordinate in the vicinity of spin crossing points; and $(v)$ the effect of spin state changes on reaction selectivities.
\end{abstract}

\title{
Keywords
}

Open-shell, organometallic chemistry, pairing energy, molecular orbital theory, spin crossover reactivity, minimum energy crossing point 


\section{Introduction}

The understanding of chemical structure and bonding for compounds containing transition metals is one of the most exciting and intellectually rewarding areas in chemistry. The multiple variables at play in this area make the subject complicated and confusing to the newcomer but fascinating to the initiated. Transition metals have an intermediate electronegativity between the very electropositive metals at the left of the periodic table and the non metals, providing interactions with variable degrees of ionic and covalent character. The covalent interactions make use of the $d$ orbitals, allowing binding to fragments that provide any number of electrons from zero to ten (e.g. in $\mathrm{C}_{8} \mathrm{H}_{8}{ }^{2-}$ ) or even more if one considers multidentate ligands. The facile addition and removal of electrons (variability of oxidation states) affects not only the ionic/covalent bond character, but also changes the preferred coordination number and geometry through the variable electronic occupation of the metal orbitals. The availability of closely spaced occupied and empty orbitals on the metal center allows the synergy of bonding and back-bonding. In addition, one should not forget the possible occurrence of direct metal-metal bonds, both of the electron-rich type (i.e. with bond multiplicity $>1$ ) in mid-valent compounds, and electron-poor ones in low valent clusters. And so forth. This article is limited to the consideration of mononuclear complexes and will not deal with metal-metal bonding.

The first bonding theories for transition metal compounds focused on the ionic bonding approach (crystal field, ligand field) and were applied to the "Werner-type" coordination compounds. The systematic development of organometallic chemistry has challenged the crystal field theory and paralleled the development of important concepts such as $\pi$-acidity and back-bonding, Pauling's electroneutrality principle, and the EAN rule. Crystal field theory and considerations of ligand field strength and pairing energy (nephelauxetic effects) provide a framework of understanding for the magnetic properties, electronic transitions, and 
ligand exchange rates of Werner-type complexes. The implicit introduction of bond covalency in the related "ligand field" approach rationalizes certain trends (e.g. the spectrochemical series) but has limited utility for the low-valent organometallic complexes. The latter, in turn, are commonly analyzed using the covalent bonding (generally MO) approach which emphasizes the bond properties (i.e. strength, multiplicity, length, polarity, and so forth). Each of these independent approaches is appropriate for the corresponding class of molecules, because the Werner-type complexes are mostly used in opto-electronic applications and as magnetic materials, whereas the organometallic complexes are mostly employed for the development of new chemical reactivity (e.g. metal-directed organic synthesis) and catalysis, where a detailed understanding of the chemical bonding is of paramount importance. This dichotomy of electronic structure analyses is at least in part responsible for the essentially independent development of these two areas of coordination chemistry.

The validity of the EAN rule (commonly termed the "18-electron rule") is the consequence of the high bond covalence and the $\pi$-acidic nature of the ligands. All nine valence metal orbitals engage in bonding or back-bonding interactions and a large energetic gap ensues between the bonding and antibonging combinations, see Figure 1. Therefore, the system achieves maximum stability when all the bonding orbitals are filled and when all the antibonding orbitals are empty. Pairing energies are low for the relatively diffuse MOs that are generated in this class of compounds, thus all these electronically saturated molecules are diamagnetic. On the other hand, low covalence (small gap between bonding and antibonding orbitals) and high pairing energies invalidate the EAN rule for the Werner coordination compounds, because the more electronegative ligands yield more contracted metal orbitals as a consequence of the higher effective positive charge. The concept of pairing energy is useful to determine the magnetic ground state of these complexes (e.g. low-spin vs. high spin for octahedral geometries). 


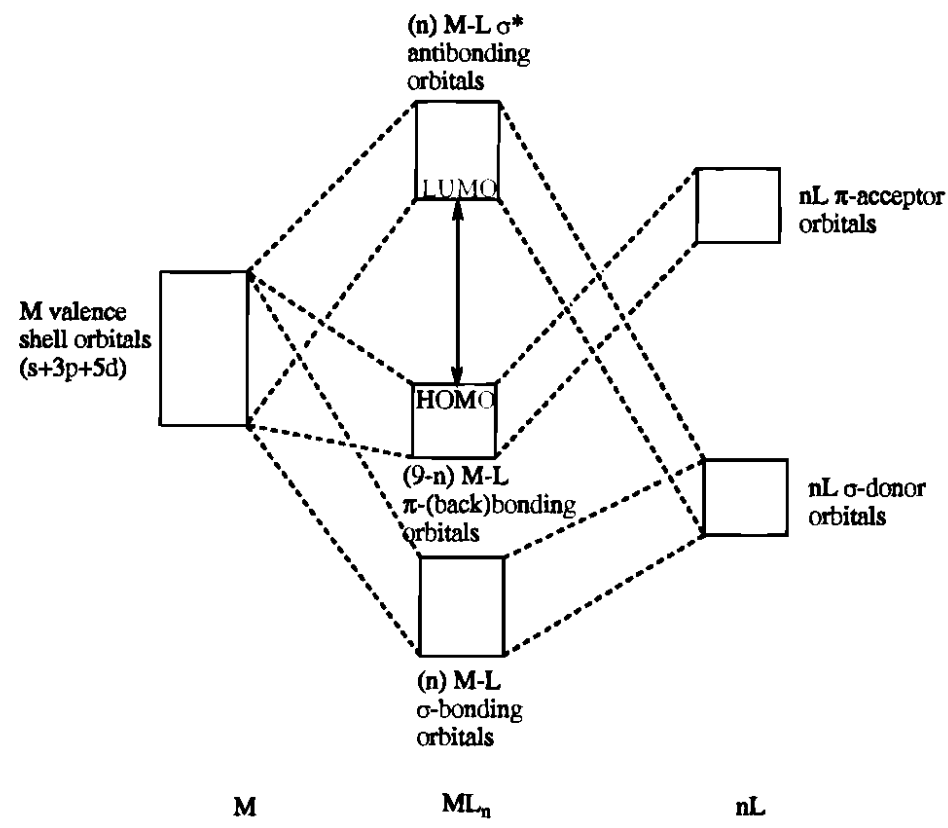

Figure 1. A generic MO correlation diagram for a low-valent, 18-electron organometallic complex. Reproduced with permission from ref. [1]. Copyright 1996 American Chemical Society.

Open-shell organometallic compounds are those compounds containing metal-carbon bonds that violate the EAN rule, i.e. they have fewer than 18-electrons in the metal valence shell. Members of this class of compounds have been known for quite some time (e.g. the 15-electron vanadocene with a spin quartet ground state was described by Fischer in 1954, closely following the discovery of ferrocene). ${ }^{[1]}$ They typically involve metals with oxidation states and coordination geometries that are reminiscent of the Werner complexes. They show, at the same time, interesting spectroscopic and magnetic phenomena like the Werner complexes and chemical reactivity akin to the lower-valent electronically saturated analogues. The development of this area, however, has been slow and these molecules have long remained at the edge of interest in both main streams of coordination chemistry research, namely Werner-type coordination chemistry and organometallic chemistry. They can be seen, in fact, as bridging the gap between these two classical areas of coordination chemistry and a framework of understanding has been developed recently. ${ }^{[2]}$ This rather 
simple approach allows a unified view of coordination chemistry as a whole and, in addition, allows the rationalization of much experimental work and the prediction of new chemistry. Elements of this approach are now starting to be included in textbooks. ${ }^{[3]}$

This article summarizes the basic framework for this unified view and the reader is referred to the original article $^{[2]}$ for a more detailed description and for a comprehensive review of the open-shell organometallic chemistry literature up to 1996 . However, the application of this framework has led to the development of additional new concepts ${ }^{[4,5]}$ that warrant the renewed description and further elaboration presented herein. Selected examples that help illustrate these concepts will be drawn from the recent literature, with an inevitable bias in favor of our own work. It is also important, however, to underline the pioneering work of Schwarz, Shaik and co-workers in the interpretation of gas phase reactivity involving highly unsaturated fragments and for the introduction of the "Two-State Reactivity" (TSR) paradigm. ${ }^{[6,7]}$ This work has strongly contributed to alert the chemical community on the possible involvement of higher-spin intermediates and spin crossover phenomena in organometallic reactions.

\section{Steric and electronic protection of unsaturation}

In order to rationalize the stability of open-shell organometallic compounds and their inability to reach a more saturated configuration in the presence of additional ligands, two effects are commonly invoked: excessive steric encumbering and electronic protection. The steric effect can be quantitatively associated to the cone angle. ${ }^{[8]}$ The achievement of a saturated configuration is impossible because interligand van der Waals repulsions exceed the stabilization energy provided by the new bond(s) being formed. Examples illustrating this case are the tris(trimethylsilyl)methyl ligand in $\mathrm{M}\left[\mathrm{C}\left(\mathrm{SiMe}_{3}\right)_{3}\right]_{2}(\text { e.g. } \mathrm{M}=\mathrm{Mn})^{[9]}$ and the norbornyl ligand in $\mathrm{M}(\text { norbornyl })_{4}($ e.g. $\mathrm{M}=\mathrm{Cr}, \mathrm{Mn}, \mathrm{Fe}, \mathrm{Co}),{ }^{[10]}$ whereas smaller alkyl 
ligands lead to more saturated structures containing additional neutral donors. The stability of the 17-electron $\mathrm{V}(\mathrm{CO})_{6}$ compound can also be attributed to steric protection, the process of relevance here being dimerization with $\mathrm{V}-\mathrm{V}$ bond formation. The second argument, electronic protection, has two different facets as shown in Figure 2.

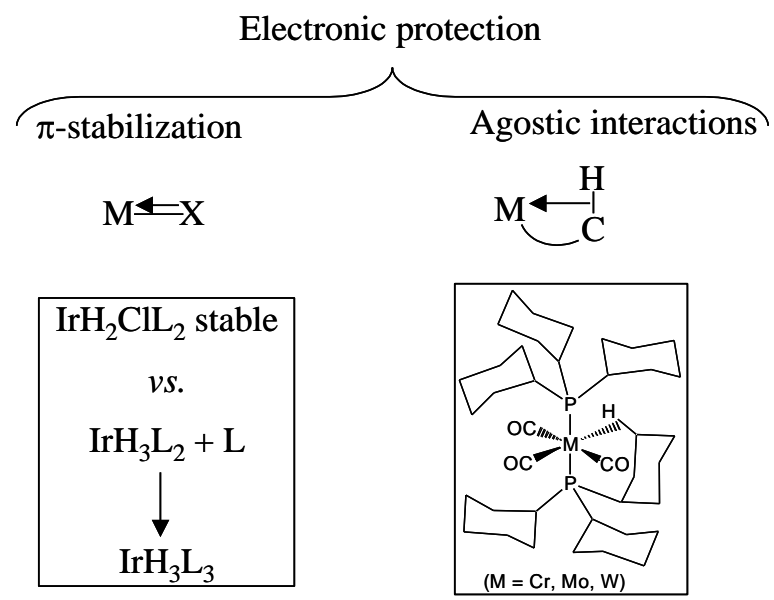

Figure 2. Two different ways in which ligands may provide electronic protection to unsaturated organometallics.

The first one, $\pi$ stabilization, plays a role when a donor atom that is already $\sigma$-bonded to the metal center has at least one additional lone pair at its disposal. These additional electrons can then be made available to the metal via a bonding interaction of $\pi$ symmetry that will effectively saturate the metal center. ${ }^{[11]}$ An example to illustrate this point is the comparison between the stable 16 -electron $\mathrm{IrH}_{2} \mathrm{ClL}_{2}(\mathrm{~L}=$ tertiary phosphine $),{ }^{[12]}$ and the non existence of the corresponding $\mathrm{IrH}_{3} \mathrm{~L}_{2}$ where none of the ligands possesses a lone pair. ${ }^{[13]}$ The second facet, agostic interactions, typically involves $\mathrm{C}-\mathrm{H}$ bonds (but sometimes also other kinds of bonds) in the vicinity of a donor atom, usually in the $\beta$ position, as shown for instance by complex $\mathrm{Cr}(\mathrm{CO})_{3}\left(\mathrm{PCy}_{3}\right)_{2} \cdot{ }^{[14]} \quad$ The $\mathrm{C}-\mathrm{H}$ bonding electrons are made available to the unsaturated metal center in "3-center-2-electron bonds". It should be noted that, in a formal sense, these $\sigma$-bonding electrons are donated to the metal center through a bonding interaction of $\sigma$ symmetry and should therefore be counted as part of the EAN. However, 
these interactions are weak, difficult to unambiguously establish experimentally, and can often be broken and reformed reversibly in rapid dynamic intramolecular processes in solution. Like the M-L $\pi$ interactions, they may be considered as an added stabilizing factor for the open-shell configuration. Both $M-L \pi$ interactions and agostic interactions are generally weaker than the M-L $\sigma$ bonds of additional ligands. Therefore, unencumbered coordination spheres will allow the hosting of new ligands to replace them, whereas more encumbered coordination spheres will not. This means that a certain degree of interplay exists between steric and electronic stabilization.

\section{Release of pairing energy as a stabilizing factor for open shell organometallics}

The stability of certain open-shell organometallic complexes cannot be rationalized solely on the basis of the two effects described in the previous section. In such cases, one always observes the presence of more than one unpaired electrons $(S>1 / 2)$. As an illustrative example, let us consider the isoelectronic $d^{3}$ half-sandwich systems $\mathrm{CpVXL}_{2}$ and $\mathrm{CpCrX}_{2} \mathrm{~L}(15$-electron configuration, $\mathrm{S}=3 / 2){ }^{[15,16]}$ These complexes are stable also when $\mathrm{X}$ and L do not have additional lone pairs (e.g. alkyls and phosphines, respectively) and their structural analysis does not reveal agostic bonds. The steric capability of the systems to accommodate an additional ligand is demonstrated by the stability of four-legged piano stool structures for the related $d^{2} \mathrm{CpVX}_{2} \mathrm{~L}_{2}$ (16-electron) and $d^{4} \mathrm{CpCrXL}_{3}$ (18-electrons). Other good examples are provided by $d^{4}$ complexes $\left[\mathrm{CrR}_{4}\right]^{2-}(12 \text {-electrons, } \mathrm{S}=2)^{[17,18]}$ and by $d^{5}$ complexes $\left[\mathrm{MnR}_{4}\right]^{2-}(13$-electron, $\mathrm{S}=5 / 2),{ }^{[19,20]}$ even for $\mathrm{R}$ as small as Me. The reason for the relative stability of these systems can be explained by invoking the concept of electron pairing energy that is so very familiar to the Werner coordination chemist.

Let us take the example of a saturated (18-electron) complex to which a neutral ligand $\mathrm{L}$ is removed to yield a 16-electron complex. Let us further imagine that at least one metal- 
based lone pair is available (e.g. configuration $d^{\mathrm{n}}$ with $\mathrm{n} \geq 2$ ). The bond being broken is represented in Figure 3 by a bonding orbital which is related by dotted lines to the ligand lone pair and to the metal accepting orbital in the 16-electron fragment (empty coordination site). Contrary to the saturated precursor, the HOMO-LUMO gap $(\Delta \mathrm{E})$ in the less saturated complex is potentially very small. Thus, if the pairing energy exceeds $\Delta \mathrm{E}$, the system will prefer to adopt a spin unpaired configuration.

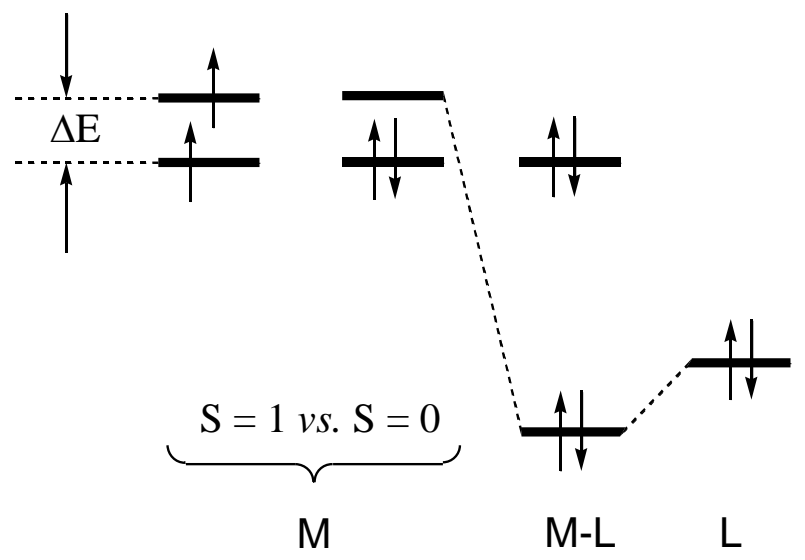

Figure 3. MO diagram for the M-L bond breaking in a 18-electron complex.

The same dichotomy can be expected upon breaking a bond in a 17-electron complex of a $d^{\mathrm{n}}$ metal with $\mathrm{n} \geq 3$ (Figure 4 ). A more exhaustive list of spin state possibilities for openshell organometallics can be found in ref. [2]. In general, whenever a coordination site (vacant orbital) is created and a metal-based lone pair is available, one may expect a spin state dichotomy depending on the relative importance of a pairing energy and an orbital splitting.

The ligand dissociation processes outlined above can be viewed along the reaction coordinate as shown in Figure 5, highlighting four distinct cases. In case (a), the product generated by ligand dissociation is more stable in the same low spin configuration as the starting complex, thus any spin related issue can be neglected in a thermally induced reaction. 
In case (b), a greater pairing energy leads the less saturated product to prefer a higher spin configuration. Thus, the product is stabilized by unpairing two electrons and this form of energetic stabilization of open shell structures can be associated to the release of pairing energy. This concept has been used to rationalize the "unusually weak" M-CO bonds in $\mathrm{Cp}_{2} \mathrm{VI}(\mathrm{CO})^{[21]}$ and in $\mathrm{Cp}_{2} \mathrm{Cr}(\mathrm{CO}) .{ }^{[22]}$ If this energetic gain is larger than the necessary energy to break the bond along the starting spin state surface, we have situation (c). In this case, the system is stable with a less saturated configuration because it would cost more energy to pair two electrons (thereby creating the necessary vacant orbital for the new bond) than the energetic gain resulting from the bond formation in the lower spin state. This unsaturated system may be forced to pair the electrons and form a new bond by adding a ligand capable of forming a stronger M-L bond, in which case we fall back to situation (b). In the extreme case (d), the system always remains in the higher spin state. The latter situation has been highlighted for the associative phosphine exchange process on the spin quartet $\mathrm{CpCrCl}_{2}\left(\mathrm{PR}_{3}\right)$ system. ${ }^{[23]}$ Cases (b) and (c) are particularly interesting because they involve a change of spin state along the reaction coordinate, leading to the general phenomenon of spin crossover reactivity.

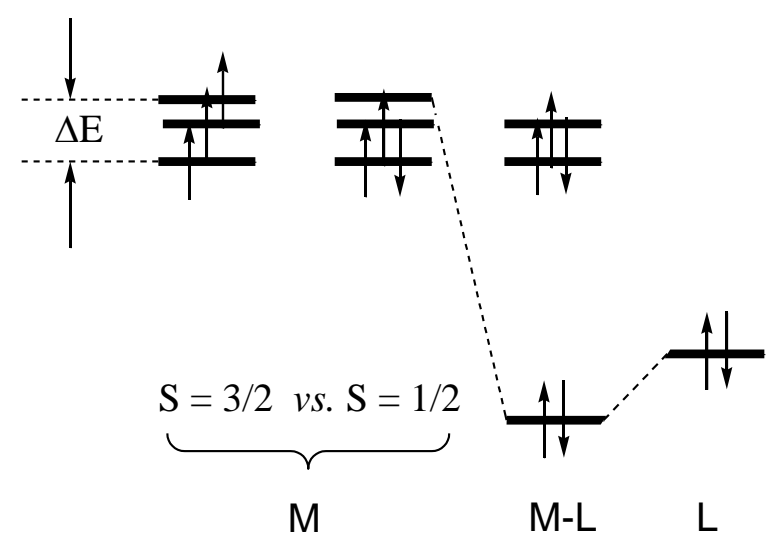

Figure 4. MO diagram for the M-L bond breaking in a 17-electron complex. 
(a)

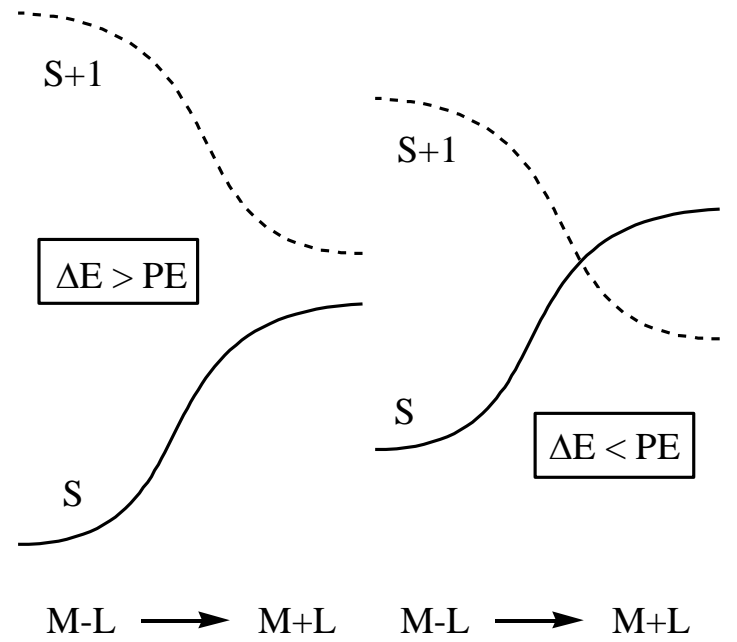

(b)

(c)

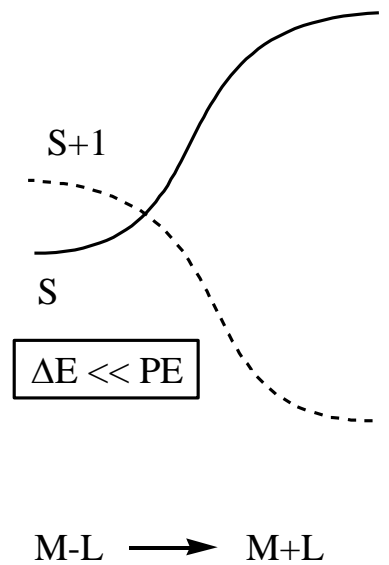

(d)

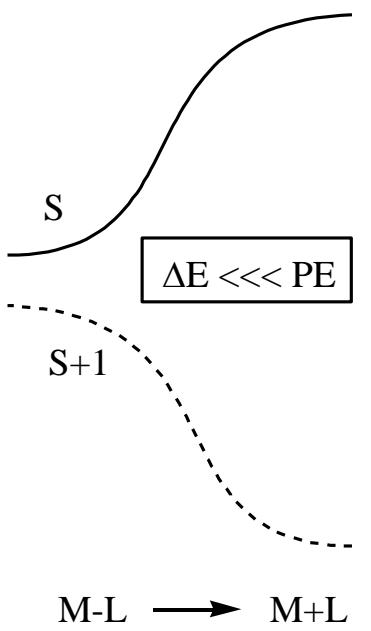

Figure 5 Possible reaction coordinates for a ligand dissociation reaction.

It should be emphasized that the four possible situations illustrated in Figure 5 relate not only to ligand dissociation processes, but also to any fundamental process leading to the generation or disappearance of one open coordination site (e.g. reductive elimination, migratory insertion, etc. and their reverse processes). The bond strength considerations used above for the ligand dissociation process must be adjusted for the other fundamental processes, for instance the reductive elimination involves breaking two bonds and forming a third one. This concept of spin crossover reactivity has far-reaching implications in organometallic chemistry, as all catalytic activities depend upon the availability of open coordination sites in one or more intermediates around the catalytic cycle.

It is possible to qualitatively predict the relative importance of pairing energy for establishing open-shell configurations and to understand observed trends of stability and reactivity of open-shell organometallics. ${ }^{[2]}$ The pairing energy increases with the formal oxidation state for a given metal atom, because a higher positive charge density has the effect of contracting the metal orbitals and it costs more energy to pair electrons in a smaller orbital because of greater Coulombic repulsion. Indeed, open-shell configurations are more 
common in higher oxidation states, whereas low-valent complexes follow more systematically the 18-electron rule. By the same token, more electron donating ligands will reduce the pairing energy and more electron withdrawing ones will increase it. For instance, the diamagnetism of $\operatorname{Ti}\left(\mathrm{CH}_{3}\right)_{2}(\mathrm{dmpe})_{2}$ and the paramagnetism $(\mathrm{S}=1)$ of $\mathrm{TiCl}_{2}(\mathrm{dmpe})_{2}$ were attributed to the greater electronegativity of $\mathrm{Cl} v s . \mathrm{CH}_{3}{ }^{[24]}$ Within the same oxidation state and coordination sphere, the pairing energy decreases upon descending from the first row to the two lower rows, because the $3 d$ orbitals are less diffuse than the $4 d$ and $5 d$ orbitals. An example of this phenomenon is the reluctance of 15 -electron $\mathrm{CpCrX}_{2} \mathrm{~L}$ compounds $(\mathrm{S}=3 / 2)$ to add an extra ligand, whereas Mo forms a wide variety of 17 -electron $\mathrm{CpMoX}_{2} \mathrm{~L}_{2}$ complexes $(S=1 / 2) .{ }^{[25]}$ For tungsten, another factor also plays a role, namely the greater strength of metal-metal bonds, leading to electronically saturated, dinuclear structures. ${ }^{[26]}$ Achieving a 17 -electron configuration for $\mathrm{CpCr}(\mathrm{III})$ requires the use of the $\pi$-acidic, highly nephelauxetic isocyanide ligands, ${ }^{[27]}$ whereas the rare examples of 15 -electron organometallic Mo(III) and W(III) complexes are highly sterically protected. ${ }^{[28-30]}$

It is generally difficult, on the other hand, to predict trends in orbital splitting since these are specific for each coordination geometry, in addition to varying with ligand strength and metal nature. In Werner-type octahedral complexes, the orbital splitting that determines the spin state ( $\Delta_{\mathrm{O}}$ between the $t_{2 g}$ and $e_{g}$ orbitals) increases with the atomic number in a Group of transition metals (e.g. $\mathrm{Fe}<\mathrm{Ru}<\mathrm{Os}$ ), because the $t_{2 g}$ orbitals are nonbonding or $\pi$-bonding whereas the $e_{g}$ orbitals are M-L $\sigma$ antibonding and thereby destabilized to a greater extent by the stronger covalent bonding in the lower row metal complexes. On the other hand, both orbitals competing for hosting the electrons for open-shell organometallic complexes are non bonding or slightly $\pi$ bonding. Further work in this area is desirable. It is important to emphasize that this analysis of spin state preference in terms of the competition between an orbital splitting and a pairing energy follows the qualitative way of thinking that is very 
familiar to the coordination chemist, with its advantages and disadvantages: it is based on visually simple concepts and allows some degree of prediction but is not quantomechanically rigorous.

All the above analyzed stabilizing factors (steric, $\pi$-bonding, agostic bonding, and release of pairing energy) are not mutually exclusive; they can simultaneously impart stability to an open-shell organometallic compound. This situation can be qualitatively illustrated by the $\left(\mathrm{C}_{5} \mathrm{R}_{5}\right) \mathrm{MoCl}\left(\mathrm{PR}_{3}\right)_{2}$ system. A role for steric protection is suggested by the reluctance of $\mathrm{CpMoCl}\left(\mathrm{PMe}_{3}\right)_{3}$ to dissociate a phosphine ligand, by the phosphine dissociation equilibrium between $\mathrm{CpMoCl}\left(\mathrm{PMe}_{2} \mathrm{Ph}\right)_{3}$ and electronically unsaturated $\mathrm{CpMoCl}\left(\mathrm{PMe}_{2} \mathrm{Ph}\right)_{2}$, and by the stability of $\mathrm{Cp} * \mathrm{MoCl}\left(\mathrm{PMe}_{3}\right)_{2 .}{ }^{[31]}$ A role for electronic protection by the chlorine lone pairs in the same open-shell compounds is suggested by the non existence of the corresponding hydride species. Finally, since these are spin triplet complexes, a pairing energy-related stabilization must also be present. A theoretical approach for the estimation of the relative importance of these three factors has been elaborated. ${ }^{[32]}$ It is interesting to make a parallel, using the VB approach, between this "16-electron" triplet $\left(\mathrm{C}_{5} \mathrm{R}_{5}\right) \mathrm{MoCl}\left(\mathrm{PR}_{3}\right)_{2}$ system and triplet dioxygen: besides the $\sigma$ bond, the metal and chlorine atoms establish two threeelectron $\pi$ interactions, thereby providing a Cl-to-Mo $\pi$-donation that is worth two electrons overall, an effectively saturated (18-electron) configuration, and a $\pi$-antibonding character for the two unpaired electrons, see Figure 6. For dioxygen, the two three-electron interactions are established in a symmetric fashion, satisfy the octet rule for both oxygen atoms, and equally yield two $\pi$-antibonding unpaired electrons. 

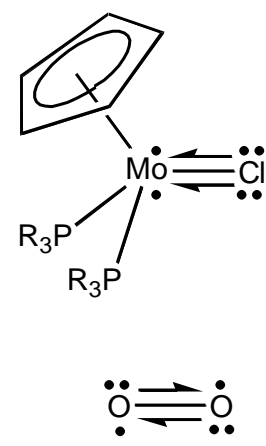

Figure 6. Bonding equivalence between $\pi$-stabilized multiple bonds in triplet molecules.

\section{Spin state as a controlling factor in chemical reactivity}

As shown in the previous sections, the spin state of an open-shell compound of general formula $\mathrm{L}_{\mathrm{n}} \mathrm{M}-\mathrm{X}$ can be delicately tuned by the nature of the coordination environment (e.g. the ligands $L_{n}$ ) and/or the metal. In turn, the spin state may have a dramatic effect on the chemical reactivity in other parts of the coordination sphere (e.g. the M-X bond).

This situation is illustrated by the half-sandwich nitrosyl derivatives of the Group 6 metals. While Mo and $\mathrm{W}$ form stable diamagnetic $\mathrm{CpM}(\mathrm{NO}) \mathrm{Cl}_{2}$ complexes, the related $\mathrm{Cr}$ compound is unstable. Attempts to generate this molecule by oxidation of the stable anion $\left[\mathrm{CpCr}(\mathrm{NO}) \mathrm{Cl}_{2}\right]^{-}$leads to $\mathrm{NO}$ dissociation, see Equation $1 .^{[33]}$ The reaction is completed by capture of the released $\mathrm{NO}$ by additional starting material, to yield $\mathrm{CpCr}(\mathrm{NO})_{2} \mathrm{Cl}$.

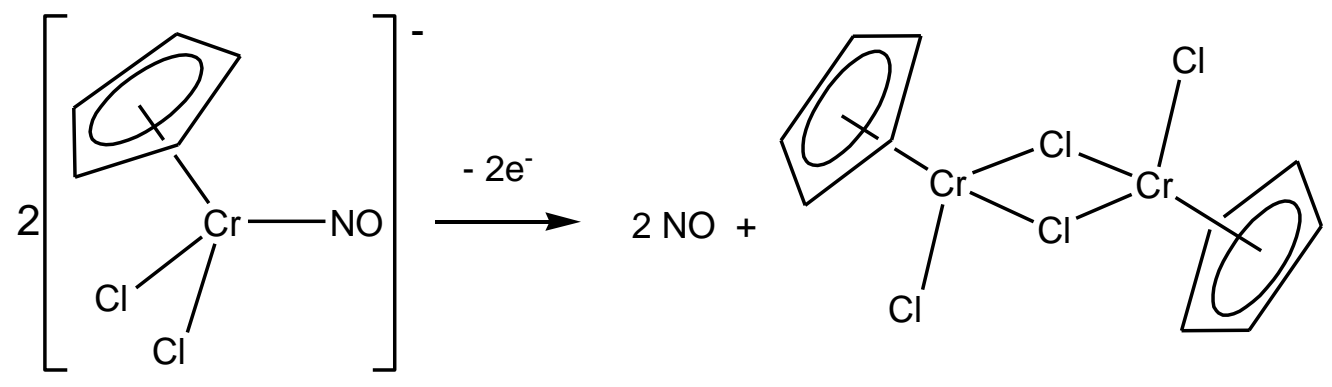


The origin of this reactivity has been traced to the preference of a spin state triplet for the putative $\mathrm{CpCr}(\mathrm{NO}) \mathrm{Cl}_{2}$ complex and to the consequent labilization of the $\mathrm{Cr}-\mathrm{NO}$ interaction, since one of the two half-occupied orbitals has a Cr-NO $\pi^{*}$ character. $^{[34]}$ The reaction is also thermodynamically favored by the stability of the spin quartet state for the resulting half-sandwich $\mathrm{Cr}(\mathrm{III})$ product, whereas the related $\mathrm{Mo}(\mathrm{III})$ and $\mathrm{W}$ (III) systems would prefer a spin doublet 17-electron configuration (see previous section). DFT calculations have shown that the spin state in $\mathrm{CpCr}(\mathrm{NO}) \mathrm{XY}$ molecules is tuned by the nature of the $\mathrm{X}$ and $\mathrm{Y}$ substituents through a combination of nephelauxetic effects and orbital ( $\pi$ bonding) interactions. Thus, a strongly $\pi$-donating $\mathrm{NR}_{2}$ ligand favors the singlet state through an increase of the HOMO-LUMO gap, whereas the replacement of a $\mathrm{Cl}$ by an alkyl ligand reduces the pairing energy. ${ }^{[34]}$ Indeed, $\mathrm{CpCr}(\mathrm{NO}) \mathrm{XY}$ complexes with $\mathrm{X}=$ amido and $\mathrm{Y}=$ alkyl are stable and diamagnetic.

\section{Spin crossover reactions: the forbiddenness factor}

Spin crossover reactivity has been well appreciated in chemistry for quite some time. Apart from the well developed area of photochemistry, spin crossover in thermally induced chemical reactivity has also received considerable attention. The general tendency, however, especially in the organic chemists community, is to consider spin crossover reactions as forbidden as a consequence of the spin conservation rule (e.g. see Figure 7). The most obvious example is provided by the highly exothermic combustion of hydrocarbons by ${ }^{3} \mathrm{O}_{2}$, whereas the corresponding reaction of ${ }^{1} \mathrm{O}_{2}$ is very rapid. The reason for this "forbiddenness" is that the transmission factor is proportional to the strength of the spin-orbit coupling Hamiltonian which mixes the two different spin hypersurfaces in the region of crossing. For light elements such as oxygen and carbon, the extent of spin-orbit coupling is small, resulting 
in very low transmission factors, hence a high level of "forbiddenness". This result, however, cannot be extrapolated to the transition metals.

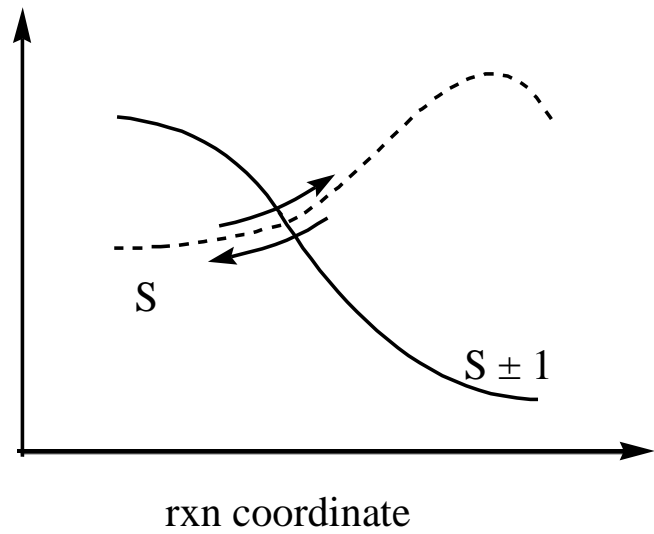

Figure 7. Reaction coordinate for a spin-forbidden two-state reaction, such as a hydrocarbon combustion with ${ }^{3} \mathrm{O}_{2}$.

When the electrons involved in the spin flip are highly localized on the transition metal atom, the greater strength of the spin-orbit coupling interaction provides high transmission factors, effectively removing the forbidden nature of the reactions. The reaction coordinates can therefore be better imagined as adiabatic surfaces where the spin state smoothly changes from that of the reagents to that of the products through a continuum of mixed spin situations around the transition state (avoided crossing), as shown in Figure 8. The electronic spin is no longer a good quantum number for the system.

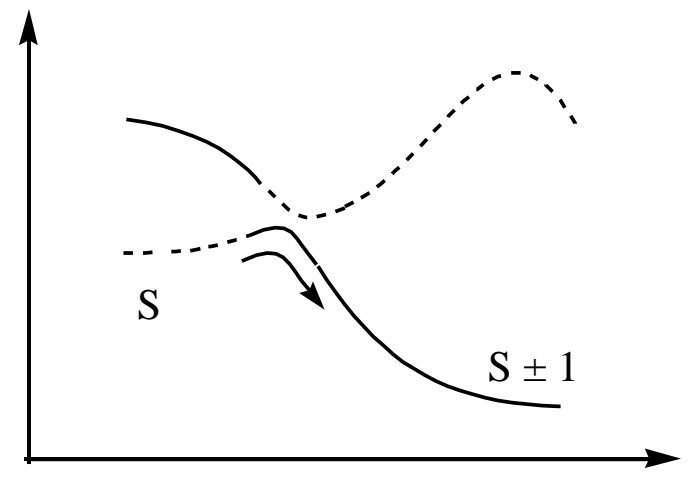

rxn coordinate 
Figure 8. Reaction coordinate for an exothermic two-state reaction with a high transmission factor and a spin crossover transition state.

The importance of spin state changes in organometallic reactivity has first been emphasized in a series of studies devoted to the gas phase reactivity of metal atoms and other highly unsaturated fragments, therefore generally containing several unpaired electrons, with small molecules including hydrocarbons. ${ }^{[6,35]}$ The relevance pf this phenomenon to biological chemistry (e.g. the $\mathrm{C}-\mathrm{H}$ bond oxidation by cytochrome $\mathrm{P}-450)^{[7]}$ has been highlighted. The pioneering work of Weitz on the gas-phase reactivity of spin triplet $\mathrm{Fe}(\mathrm{CO})_{4}$ should also be underlined. ${ }^{[36-38]}$ We emphasize in this article, however, that spin crossover reactivity is a common phenomenon also for reactions of commonly available organometallic compounds in solution. The term "Two-State Reactivity" that has been introduced to describe this phenomenon is, at the same time, more general and more limitative that the term "Spin Crossover Reactivity". It is more general because it may also be applied to avoided crossings between two surfaces with the same spin but different space symmetry. This situation may also lead to significant barriers, as shown recently for certain atom transfer

reactions. ${ }^{[39,40]}$ It is more limitative because more than two spin states may be involved in some cases. However, a spin crossover point will generally involve only two spin surfaces, as simultaneous crossing of three surfaces into a single point in space will be a rare occurrence, much like three-particle collisions are a rare event with respect to two-particle collisions.

\section{Minimum energy crossing points}

A reaction occurring entirely in one spin state follows a reaction coordinate that can be traced on a singe potential energy surface (PES). This is a hypersurface in configuration space, i.e. the space identified by the free energy against the $3 \mathrm{~N}-6$ internal coordinates or 
normal modes of the system. The reaction rate is related to the highest free energy transition state, which is a stationary point on the PES, or Saddle Point (SP). That is, all 3N-6 partial derivatives of the free energy along the normal modes are zero, while all second derivatives are positive except along the normal mode that corresponds to the reaction coordinate (imaginary vibrational frequency). For a reaction involving a spin state change, the system jumps from one PES to another one of different spin (in the diabatic approximation), which can in principle occur at any point where these two surfaces cross. The intersection or seam of crossing between two PESs with 3N-6 dimensions is a hyperline, which defines a 3N-7dimensional space. Any point in this space corresponds to a situation where the geometry and the energy of the system is identical in both spin state, thus satisfying the Franck-Condon principle for spin crossing. The most convenient point where the system may cross is the point at minimum energy within this space, which is called Minimum Energy Crossing Point or MECP. Note that MECPs are not stationary points on either PES, but are stationary points in the 3N-7-dimensional seam of crossing. Algorithms have been developed for the calculation of MECPs. ${ }^{[41-45]}$

As shown in the previous section, the spin-orbit coupling Hamiltonian will generate a surface mixing resulting in an avoided crossing. From the computational viewpoint, however, a convenient method for solving the Schrödinger equation with the inclusion of the spin-orbit coupling term is not available. It is therefore more convenient to focus on the intersection between the two adiabatic spin surfaces. The error is usually confined within a few hundred $\mathrm{cm}^{-1}$, depending on the strength of the spin-orbit coupling. ${ }^{[4]}$

From the computational perspective, MECP calculations are actually rather inexpensive and less ambiguous when compared to alternative methods for the location of the region in configuration space where spin crossover occurs. An alternative method that has often been employed consists in identifying one "pivot" internal parameter that best describes the reaction coordinate (it exhibits the largest variation), keeping it fixed at a number of different 
values between those found in the reagent and product, and optimising all other parameters at each value and in each spin state. As an example, consider the dissociation of a $\mathrm{PMe}_{3}$ ligand from spin doublet $\mathrm{CpMoCl}_{2}\left(\mathrm{PMe}_{3}\right)_{2}$ to yield spin quartet $\mathrm{CpMoCl}\left(\mathrm{PMe}_{3}\right)$. The best parameter in such case is the Mo-P distance of the bond being broken. This "partial optimisation" method yields the results shown in Figure 9. ${ }^{[46]}$ The point where the two lines cross is not the MECP but rather a lower estimate of it, since only one parameter (the Mo-P distance) is the same for the two independently optimised structures. If we now calculate the two structures of the lower estimate in the other spin state, we obtain the so-called "vertical" excitation energy for each structure. The lower of these two energies gives an upper estimate of the MECP, thus a bracket is available where the MECP energy may be placed. For the example shown in Figure 9, the upper estimate lies above the energy of the asymptote of the spin doublet dissociation curve. Therefore, an ambiguity remains in this case as to whether the phosphine substitution reaction follows a spin crossover pathway or rather takes place entirely along the spin doublet surface. This ambiguity has been removed by the explicit calculation of the MECP. ${ }^{[46]}$

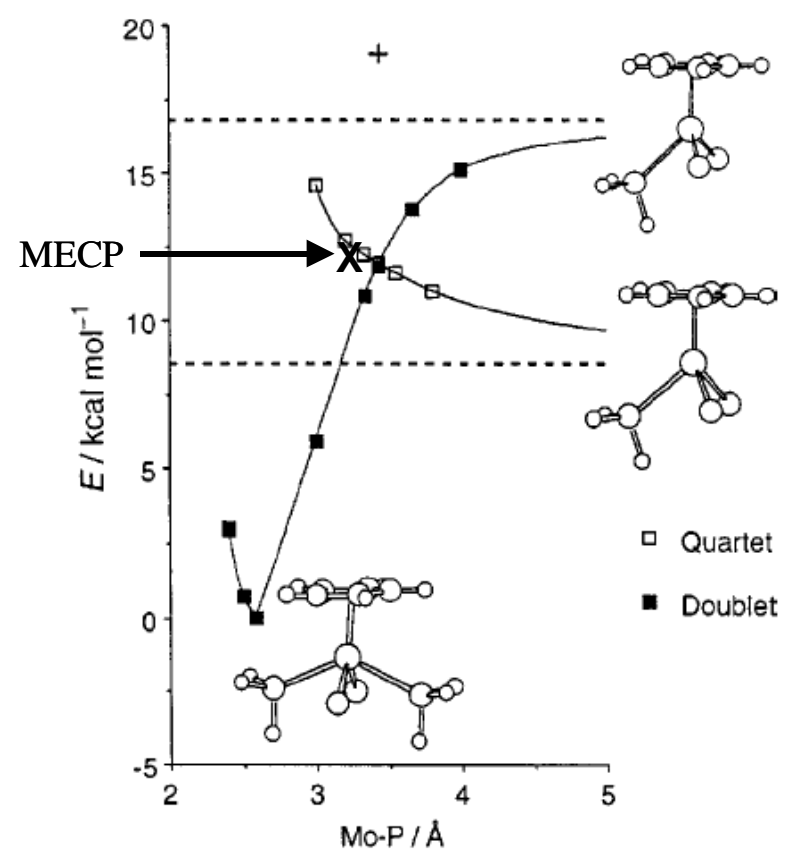


Figure 9. Energies of partially optimized $\mathrm{CpMoCl}_{2}\left(\mathrm{PH}_{3}\right)_{2}$ at fixed Mo-P distances along the doublet and quartet spin surfaces. The dashed lines show the energies of the fully dissociated systems. $(+)$ :estimated upper limit of the crossing point energy; (X): MECP. Reproduced with permission from Ref. ${ }^{[46]}$. Copyright 2000 Royal Society of Chemistry.

\section{Spin crossover vs. saddle point transition states}

We must distinguish two possible situations, depending on whether the crossing point precedes or follows the saddle point on the reagent's spin surface. Namely, crossing may occur on the upslope of the reaction coordinate (e.g. Figure 7 and Figure 8), thereby corresponding to the highest energy point of the two-state reaction pathway (TS = MECP), or on the downslope after the SP on the reagent PES (e.g. see Figure 10, in which case TS = SP). Note than in both cases the MECP energy is lower than that of the SP for the single state pathway. However, only in the first case is the reaction rate controlled by the spin crossover. In this case, it must be recognized that the need for the spin change imposes a barrier, due to the need to reach a strained configuration where the spin crossover is allowed by the Franck-Condon principle. This is an enthalpic barrier. It has nothing to do with the transmission factor (forbiddenness), which finds itself incorporated into the entropic term of the activation free energy. When, on the other hand, the MECP follows the SP, the factors controlling the reaction rate are the same as for any single-state reaction. In such cases, the traditional transition state theory and concepts such as Hammond's principle may be applied.

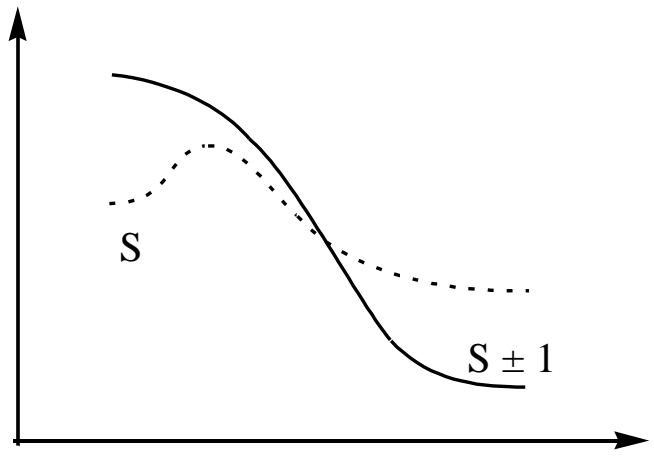

rxn coordinate 
Figure 10. Reaction coordinate for a two-state reaction with a one-state transition state.

Experimentally, it may be quite difficult to determine whether a reaction rate is controlled by a spin crossover or by a saddle point. In fortunate cases, odd reaction rate trends may be useful indicators for the occurrence of a spin crossover TS, as will be shown for a few examples in the next section, but the proposition is best comforted by the computational analyses of the SP and MECP, and where they are located along the reaction coordinate. A fine example of this approach is the computational analysis of the $\mathrm{C}_{2} \mathrm{H}_{4}$ epoxidation by $\mathrm{CpCrOCl}_{2}$, a spin doublet $\mathrm{Cr}(\mathrm{V})$ complex, leading to $\mathrm{CpCr}\left(\mathrm{OC}_{2} \mathrm{H}_{4}\right) \mathrm{Cl}_{2}$, a spin quartet $\mathrm{Cr}$ (III) complex. ${ }^{[47]}$ The reaction rate is limited by the rupture of the $\mathrm{C}-\mathrm{C}$ and $\mathrm{Cr}-\mathrm{O} \pi$ bonds, accompanied by the formation of the O-C $\sigma$ bond, along the doublet PES, see Figure 11, while the MECP is located at a later point, near the doublet intermediate which features a carbon centered radical, spin-opposed to two unpaired electrons on the formally $\mathrm{Cr}(\mathrm{IV}), d^{2}$ center. Thus, the spin crossover does not influence the reaction rate. It should be noted that this reaction involves spin unpairing, e.g. it is topologically related to the ligand dissociation process in Figure 5(c).

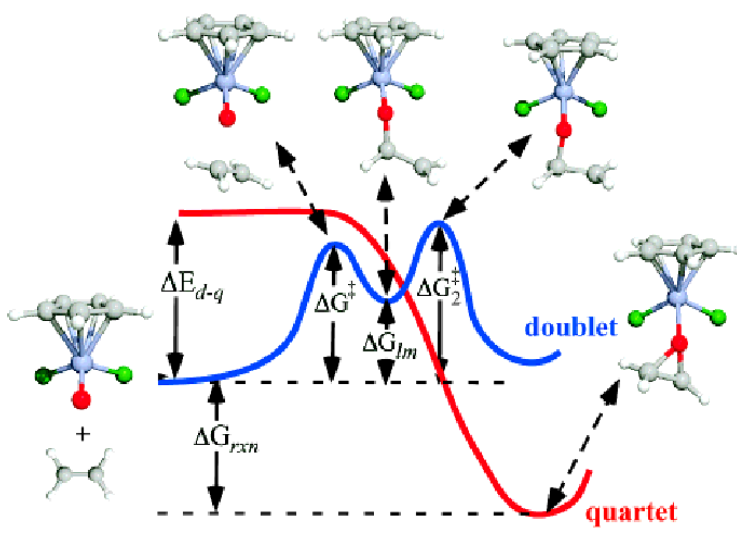

Figure 11. Reaction coordinate for the ethylene epoxidation by $\mathrm{CpCrOCl2}$. Reproduced with permission from ref. ${ }^{[4]}$. Copyright 2002 American Chemical Society.

\section{Reactions with electron pairing: influence of the coordination sphere}


We examine here the general situation of a reaction that involves an increase of the number of valence electrons by two units (e.g. ligand addition, oxidative addition, the reverse of migratory insertion, etc.) for an open-shell system where all potentially available empty coordination sites (metal-based orbitals) contain one unpaired electron. Thus, a spin change must occur to allow the reaction, see Figure 12. This situation is topologically related to the inverse of the ligand dissociation process in Figure 5(b).

The approach of the additional electron pair (e.g. a lone pair for a ligand addition; two $\sigma$-bonding electrons for an $\mathrm{X}-\mathrm{Y}$ oxidative addition or for the inverse of a migratory insertion; etc.) to the lower spin configuration is in principle attractive and without an energy barrier, as shown in Figure 7 and Figure 10 (solid curves). The approach to the higher spin configuration, on the other hand, may be more or less repulsive or attractive as shown for the dashed curves in the same Figures. It is the nature of this approach that determines whether the transition state is a MECP (as in Figure 7) or a SP (as in Figure 10).

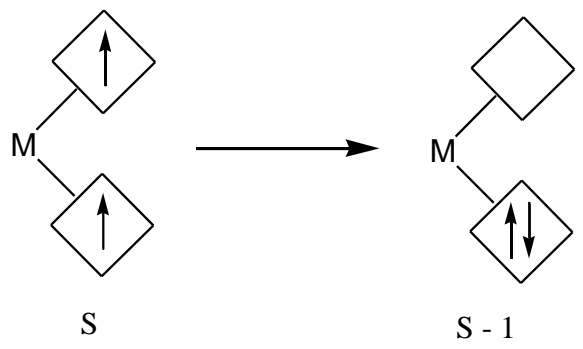

Figure 12. Generation of an open coordination site by spin pairing.

The orbital interaction between the metal in the higher spin state and the two-electron donor involves three electrons, leading to one electron being placed in an antibonding combination, while one metal-based orbital remains semi occupied. This situation will in general be energetically unfavourable. For instance, a spin triplet 16-electron complex would lead to an 18-electron complex in an excited state (one electron in a high-energy orbital, see 
Figure 1). In the absence of accompanying stabilizing effects, this approach will be repulsive leading to a spin crossover transition state.

An accompanying stabilizing effect may be the occurrence of other simultaneous processes that remove two electrons from another part of the coordination sphere (e.g ligand dissociation including ring slippage, reductive elimination, etc.). The energetic trade off between the establishment of new bonds and the weakening of old ones determines the shape of the reaction coordinate. If the new bonds being formed are stronger than those being weakened, the interaction will be an attractive one even in the higher spin state. Figure 13 shows the example of a ligand addition process that is accompanied by the weakening of another metal-ligand bond. In case of an attractive interaction, the initial M-L bond weakening leads to the crossover point on the downslope of the reaction coordinate. After the crossover point, the M-L bond can strengthen again along the lower spin PES path. The phenomenon illustrated in Figure 13 may be generalized to an oxidative addition, the inverse of a migratory insertion, etc. Analogously, the accompanying phenomenon may be generalized to other processes that lead to the removal of two electrons from the coordination sphere (e.g ring slippage, reductive elimination, etc.).

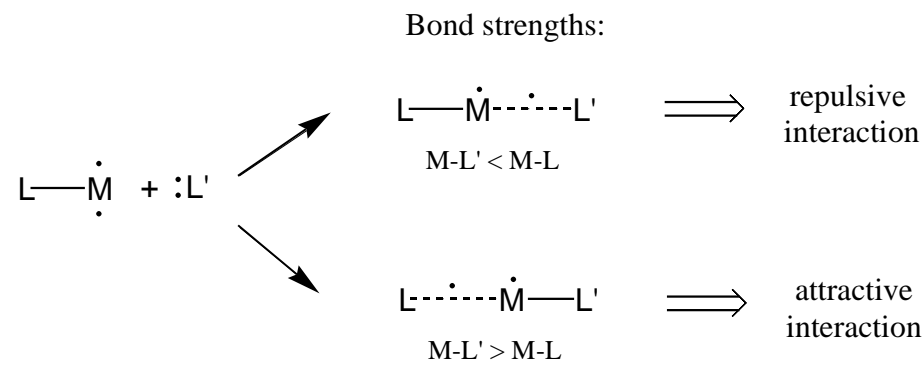

Figure 13. Ligand addition to a semi occupied accepting orbital.

This phenomenon explains the large difference in rate (three orders of magnitude) for the addition of $\mathrm{N}_{2}$ vs. $\mathrm{CO}$ to spin triplet $\mathrm{Cp} * \mathrm{MoCl}\left(\mathrm{PMe}_{3}\right)_{2}{ }^{[48]}$ This result is unusual because the two substrates are isoelectronic and isosteric and add with very similar rates to most other 
16-electron organometallic fragments, e.g. $\mathrm{CpM}(\mathrm{CO})_{3}(\mathrm{M}=\mathrm{V}, \mathrm{Nb}, \mathrm{Ta}),{ }^{[49]}$ though these are probably solvated species in the hydrocarbon or noble gas matrices in which they are photogenerated. A slightly faster $\mathrm{CO}$ addition (less than one order of magnitude) can be understood on the basis of Hammond's principle since the newly formed M-CO bond is stronger than the corresponding $\mathrm{M}-\mathrm{N}_{2}$ bond. The reason for the large rate difference for the addition to spin triplet $\mathrm{Cp} * \mathrm{MoCl}\left(\mathrm{PMe}_{3}\right)_{2}$ is the repulsive approach of $\mathrm{N}_{2}$, since this forms a bond weaker than all other already present Mo-ligand bonds, leading to a spin crossover transition state. Carbon monoxide, on the other hand, forms a much stronger bond and the initial interaction is much less repulsive. Computations show that the ligand approach is accompanied by the weakening of the Mo-Cl bond and yield MECPs whose energies are in good agreement with the experimentally measured activation barriers. ${ }^{[50]}$

Therefore, in order to predict whether a spin crossover will or will not have a retarding effect on a reaction rate involving electron pairing, it is important to evaluate not only the nature of the entering substrate and the strength of the new bonds being formed, but also the nature of the ligands already present in the coordination sphere and the type of accompanying phenomena that may occur to alleviate the energy cost of the initial repulsive approach. Thus, spin triplet $\mathrm{Fe}(\mathrm{CO})_{4}$ adds $\mathrm{Fe}(\mathrm{CO})_{5}$ much more slowly than spin singlet $\mathrm{Fe}(\mathrm{CO})_{4}$, $(3.1 \pm 0.9) \times 10^{8}$ vs. $(1.1 \pm 0.2) \times 10^{11} \mathrm{M}^{-1} \mathrm{~s}^{-1},{ }^{[36]}$ and also adds $\mathrm{CO}$ much more slowly than spin singlet $\mathrm{Ru}(\mathrm{CO})_{4}$ and $\mathrm{Os}(\mathrm{CO})_{4}$ in the addition of $\mathrm{CO}:(3.1 \pm 0.7) \times 10^{7}(\mathrm{Fe}),(1.7 \pm 0.5) \times 10^{10}(\mathrm{Ru})$ and $(3.3 \pm 0.4) \times 10^{10}(\mathrm{Os}) \mathrm{M}^{-1} \mathrm{~s}^{-1} \cdot[51,52]$ This is so because the only possible accompanying phenomenon in all these cases is weakening of a $\mathrm{M}-\mathrm{CO}$ bond, which is as strong or stronger than the new bond being formed. On the other hand, spin triplet $\mathrm{Tp} \mathrm{Co}^{\mathrm{C}}(\mathrm{CO})\left[\mathrm{Tp} \mathrm{p}^{\prime}=\right.$ hydrotris(3-isopropyl-5-methylpyrazolyl)borate] adds $\mathrm{CO}$ at close to diffusion limited rates, ${ }^{[53]}$ since the weaker Co-N bonds can temporarily weaken while the new Co-CO bond is being formed. A recent computational study confirms this view. ${ }^{[54]}$ 
Oxidative addition reactions of a X-Y substrate present another interesting phenomenon. In the initial interaction, the substrate acts as a normal L-type ligand through its $\sigma$-bonding electrons. For substrates such as $\mathrm{H}_{2}$ and the $\mathrm{C}-\mathrm{H}$ bonds of saturated hydrocarbons, this is usually a weak interaction when compared with other M-L bonds that are already present in the molecule. Thus, the interaction will in general be repulsive, leading to a spin crossover TS. Probably for this reason, the $\mathrm{H}_{2}$ oxidative addition to $\mathrm{Fe}(\mathrm{dmpe})_{2}$ is three orders or magnitude slower than the same process for $\mathrm{Ru}(\mathrm{dmpe})_{2 .}{ }^{[55,56]}$ Calculation on model systems indeed reveal a spin triplet ground state for $\mathrm{FeL}_{4}$ and a spin singlet one for $\mathrm{RuL}_{4}\left(\right.$ e.g. $\left.\mathrm{L}=\mathrm{PH}_{3}\right) .{ }^{[57]}$ Subsequently, the reaction path leads to the final oxidative addition product in the lower spin state, $[\mathrm{M}](\mathrm{X})(\mathrm{Y})$, either directly or through an intermediate, a socalled $\sigma$-complex, $[\mathrm{M}] \cdot(\mathrm{XY})$. In the latter case, there is an additional barrier for the final oxidative addition process along the lower spin PES as shown in Figure 14. The height of this barrier may be higher or lower than the initial spin crossover barrier. Thus, even when the initial interaction is repulsive, the overall process may be controlled either by the spin crossover or by the oxidative addition SP. It was shown, for instance, that the $\mathrm{CH}_{4}$ oxidative addition to triplet tungstenocene complexes to yield methyl hydride products has a higher spin crossover energy barrier for the unsubstituted $\mathrm{Cp}_{2} \mathrm{~W}$ complex and a higher oxidative addition barrier for the ansa derivative $\mathrm{CH}_{2}\left(\mathrm{C}_{5} \mathrm{H}_{4}\right)_{2} \mathrm{~W} \cdot{ }^{[58]}$ This rationalizes experimentally observed differences in rates of methane elimination $v s$. H-atom scrambling in the methyl hydride complexes.

\section{Spin acceleration}

We can imagine a wide variety of situations where a starting compound in a certain spin state (e.g. $\mathrm{S}=0$, as for most organometallic compounds) transforms into a product in the same spin state, via one (or more) open-shell intermediate(s) in a different (e.g. $\mathrm{S}=1)$ spin 
state. One simple pathway is illustrated in Figure 15. Note that the intermediate(s) may have a higher of a lower spin state, depending on whether the ongoing process is spin unpairing or pairing, corresponding to situations (b) and (c) of Figure 5. When this occurs, provided that the transmission factor is high and that the MECP between the two surfaces occurs at a lower energy than the SP on the reagents spin surface (see section 6), the reaction will be faster along the multiple state pathway than along the single state one. The rate may be controlled either by the first spin crossover process, $\operatorname{MECP(1),~or~by~the~second~one,~} \operatorname{MECP}(2)$, depending on their relative energy, but in either case both spin crossovers are at lower energy than the SP on the reagents PES. We refer to this phenomenon as a spin acceleration, as opposed to the concept of spin block which describes the spin-forbidden character of spin crossover reactions in organic chemistry.

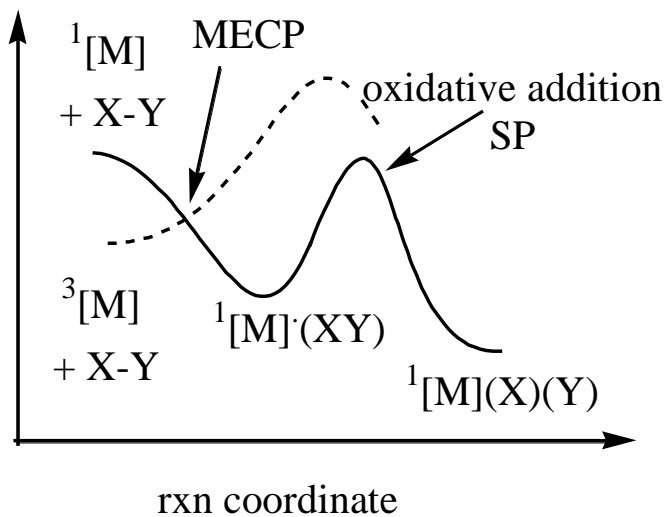

Figure 14. Generic singlet and triplet potential energy surfaces for a X-Y oxidative addition to a triplet unsaturated metal fragment, ${ }^{3}[\mathrm{M}]$.

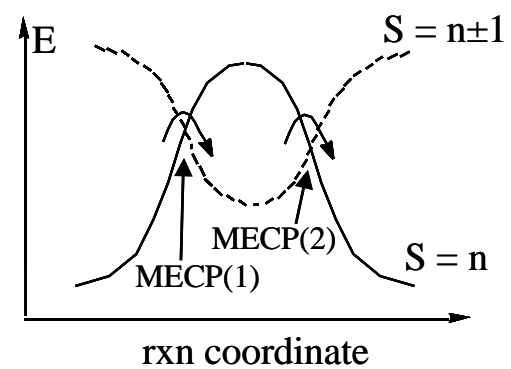

Figure 15. Reaction coordinate for a spin accelerated process of a transition metal complex. 
The most direct and unambiguous method to establish whether a reaction occurs via an intermediate with a spin state different from that of the starting material is to isolate it and to measure its magnetic properties, or to identify it by a spin selective spectroscopic method (e.g. NMR, EPR, ...) during the course of the reaction. This is, however, rarely possible because of short lifetimes, translating into small steady state concentrations. Once again, computational work helps resolve possible ambiguities.

In the application of computational methods, it is again important to underline that the explicit determination of the MECP and where it occurs along the reaction coordinate is advantageous relative to the alternative partial optimisation method. As detailed in section 6, only through the explicit MECP calculation was it possible to establish that the $\mathrm{PMe}_{3}$ exchange process on the organometallic radical $\mathrm{CpMoCl}_{2}\left(\mathrm{PMe}_{3}\right)_{2}$ has a spin crossover transition state (the MECP occurs at lower energy than the spin doublet dissociation asymptote) and is therefore spin accelerated, explaining why this process is unusually fast. ${ }^{[59]}$ The phosphine substitution reaction on $\mathrm{CpCo}\left(\mathrm{PPh}_{3}\right)_{2}$, which was equally described as unusually fast, ${ }^{[60]}$ is probably also spin accelerated, since $\mathrm{CpCoL}$ model systems have been shown to be more stable in the triplet state. ${ }^{[61]}$ One case where a spin acceleration phenomenon was suggested by the more classical partial optimisation method involves the racemization of the asymmetric 16 -electron $\mathrm{CpW}(\mathrm{CO})(\mathrm{NO})$ system. ${ }^{[62,63]}$ In this case, the chosen internal coordinate for the partial optimisation study was the fold angle $\phi$ defined by the $\mathrm{W}-\mathrm{Cp}$ (center of gravity) axis and the $\mathrm{W}(\mathrm{NO})(\mathrm{CO})$ plane, as shown in Figure $16 . \quad$ Both lower and upper estimates of the MECP are located below the SP along the reagent PES. This result may be extrapolated to other asymmetric 16-electron CpMLL' systems, many of which may be easily accessible by dissociation of a labile ligand L" from a saturated asymmetric CpMLL'L" complex. This result signifies that, contrary to initial predictions, ${ }^{[64]}$ enantiomerically pure complexes of this type may not be capable of providing high enantiomeric excesses as asymmetric Lewis-acid catalysts. 


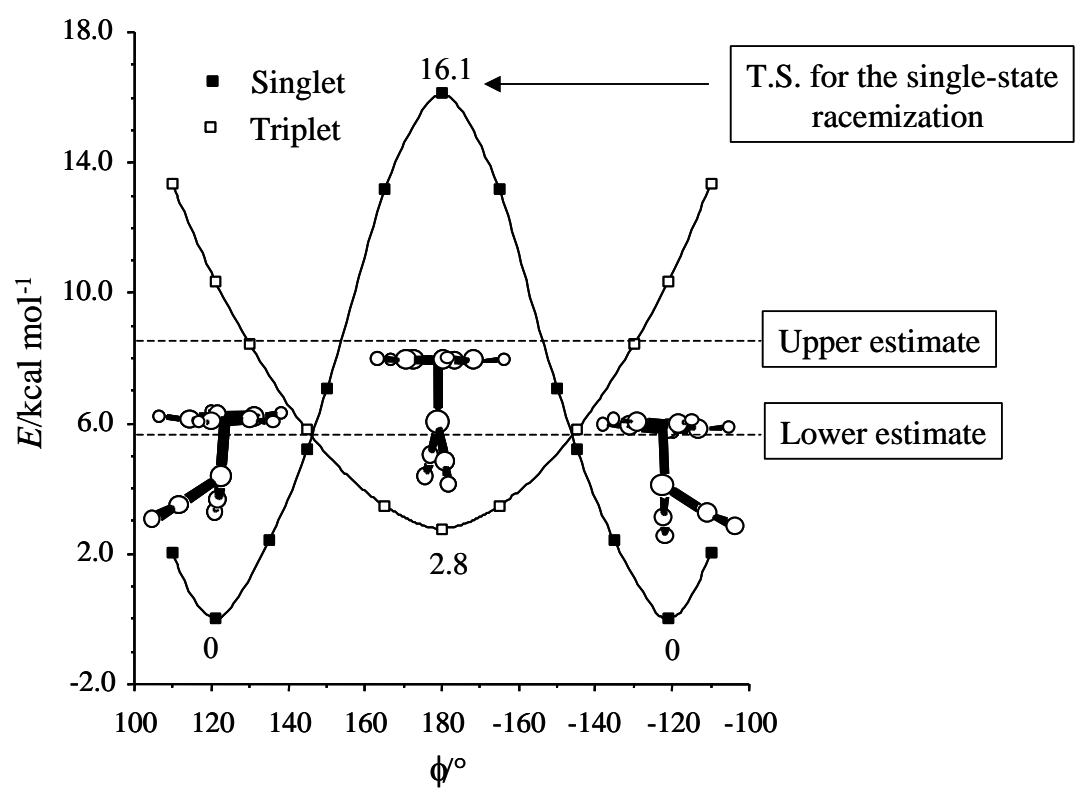

Figure 16. Spin accelerated racemization of $[\mathrm{CpW}(\mathrm{NO})(\mathrm{CO})]$. Adapted from ref. ${ }^{[63]}$.

Besides the situation involving two MECPs as described in Figure 15, it is also conceivable to imagine other situations. We shall restrict our considerations to the simpler case of a reaction that involves only one reaction intermediate, but our considerations may be easily extended to more complex situations. When the rate of generation of the intermediate is controlled by the spin crossover (the MECP precedes the reagents SP), the subsequent process may be controlled not only by an additional MECP as shown in Figure 15, but also by a SP. The latter may be located either on the intermediate PES or on the product PES, as illustrated in Figure 17 (cases $a$ and $b$, respectively). In all these cases, the reaction is still spin accelerated, provided the SP leading to the product, $\mathrm{SP}(2)$, has a lower energy than the SP on the reagent surface, $\mathrm{SP}(1)$. In addition, the different spin intermediate may be attained via a SP on the reagents PES and then transform into the products via either an MECP, a SP on the intermediate PES, or a SP on the products PES (Figure 17, cases $c, d$ and $e$, respectively). In these cases there is no spin acceleration effect, even though the reaction involves a spin crossover and an intermediate on another PES. In all cases, the rate law will depend on the relative energies of the two transition states according to the usual steady state 
approximation for the intermediate. Systems behaving in any of the fashions described in Figure 17 have not yet been identified to the best of my knowledge.

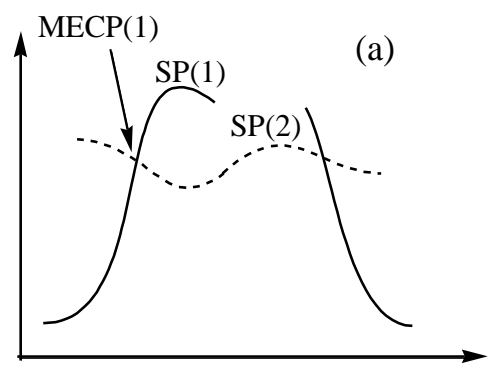

rxn coordinate

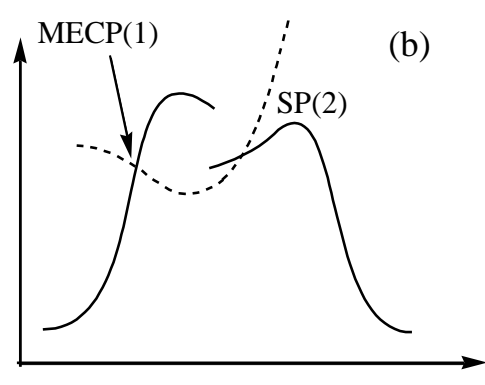

rxn coordinate

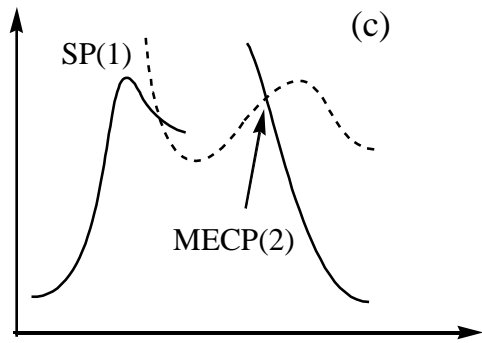

rxn coordinate

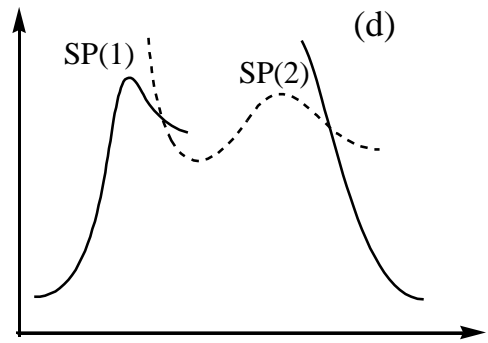

rxn coordinate

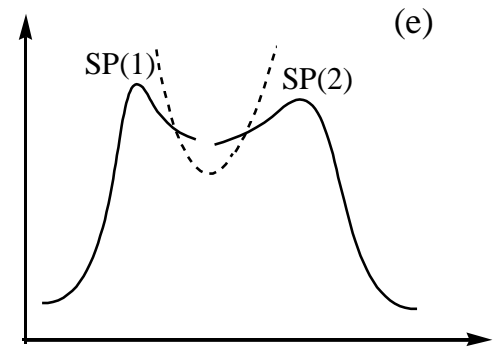

rxn coordinate

Figure 17. Different kinds of spin crossover reactions (see text).

The concept of spin acceleration has far-reaching implications in stoichiometric and catalytic reactions, thus a more clear understanding and the prediction of the ligand effects on the MECP energy will be an important goal in this area.

\section{Reaction selectivities in spin crossover reactions}

The formation of competitive products from parallel pathways is common for singlestate chemical reactions. ${ }^{[65]}$ In rare cases, the various products may be obtained through pathways that originate from the same SP, along different normal modes (this occurs for higher-order saddle points). The most common occurrence, however, is a competition between two or more pathways that have different and energetically similar saddle points, as shown in Figure 18. The relative heights of the reaction barriers and the reaction enthalpies 
determine whether the product distribution will be under kinetic or thermodynamic control at a given temperature.

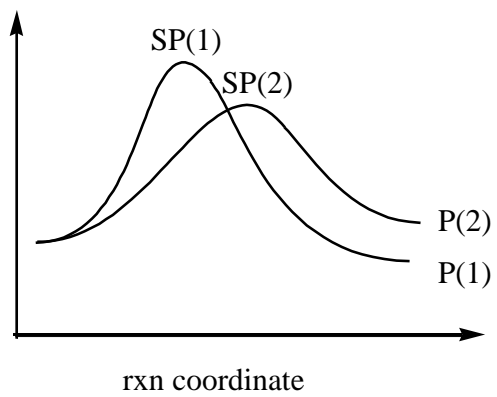

Figure 18. Competing parallel pathways leading to two products for a single-state reaction.

When a reaction involves a spin crossover, a number of possibilities may arise depending on whether all pathways are controlled by MECPs, or all by SPs, or finally some by MECPs and others by SPs. When we further consider that the kinetic competition may occur at different points along the reaction coordinate (e.g. starting from different reaction intermediates) and that these intermediate may have the same spin or different ones, it is clear that the number of possibilities is very large and hard to classify. Just to illustrate the simple example involving a discrimination between only two different pathways and where the two TSs are attained directly from the same intermediate, we can imagine the three possibilities illustrated in Figure 19. Case (a) shows a competition between two MECPs, case (b) between one MECP and one SP, and case (c) between two SPs.

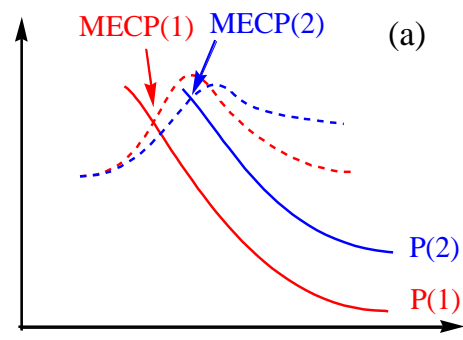

rxn coordinate

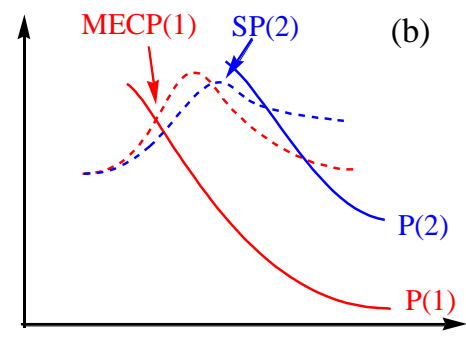

rxn coordinate

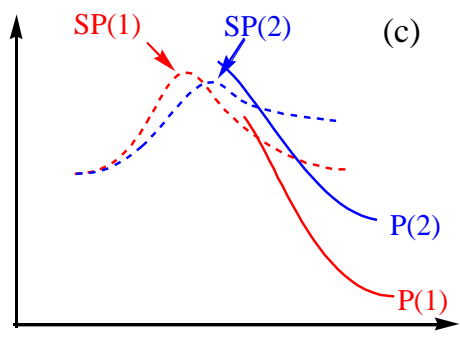

rxn coordinate

Figure 19. Competing parallel pathways leading to two products for a spin crossover reaction. 
An example of this general phenomenon was recently provided by the ethylene addition to the highly reactive 16-electron $\mathrm{Cp} * \operatorname{Ir}\left(\mathrm{PMe}_{3}\right)$ fragment, where the observed formation of the ethylene addition product, $\mathrm{Cp} * \operatorname{Ir}\left(\mathrm{PMe}_{3}\right)\left(\eta^{2}-\mathrm{C}_{2} \mathrm{H}_{4}\right)$, and of the oxidative addition product, the hydrido vinyl complex $\mathrm{Cp}^{*} \operatorname{Ir}\left(\mathrm{PMe}_{3}\right)\left(\mathrm{C}_{2} \mathrm{H}_{4}\right)(\mathrm{H}),{ }^{[66]}$ in a ca. $1: 2$ ratio could not find a proper rationalization when the consideration were limited to the spin singlet PES. ${ }^{67]}$ It is only possible to rationalize this result upon addressing the process as a spin crossover reaction. ${ }^{[68]}$ By using the simplified $\mathrm{Cp} \operatorname{Ir}\left(\mathrm{PH}_{3}\right)$ model, which has a spin triplet ground state, it was found that a common triplet intermediate, the $\sigma$-complex $\mathrm{CpIr}\left(\mathrm{PH}_{3}\right)\left(\eta^{2}-\mathrm{H}-\mathrm{C}_{2} \mathrm{H}_{3}\right)$, leads to two TSs of similar energy, $\mathrm{MECP}(1)$ and $\mathrm{SP}(1)$, see Figure 20. The first one is a spin crossover point leading to the oxidative addition product, whereas the second one is a saddle point leading to a second intermediate, a diradical $\eta^{1}$-ethylene complex with one electron localized on the carbon atom. The latter evolves further to the singlet $\eta^{2}$-ethylene product via a lower energy MRCP. Thus, the selectivity for this reaction is determined by the energetic comparison between MECP(1) and SP(1), a situation related to Figure 19(b). Incidentally, the reactive intermediate is generated by reductive elimination of cyclohexane from the diamagnetic cyclohexyl hydride, thus this is another example of a spin accelerated reaction, though according to the calculations the MECP leading to the triplet intermediate is not significantly stabilized relative to the singlet dissociation asymptote. ${ }^{[68]}$

\section{Conclusions and perspectives}

This article has attempted to address two issues. The first one is the analysis of the electronic structure and stability of coordination compounds of all kinds under the same general scheme, going from Werner complexes on one side to low valent organometallics on the other side, from the ionic bonding approach to the opposite covalent bonding approach, using all the tools that are available to the coordination chemist. Open-shell organometallics 
can be placed in the grey area within this scenario and can be viewed as a bridge between the two more traditional coordination chemistry disciplines. The second issue is the chemical reactivity of open-shell organometallics. All the fundamental reactions encountered in organometallic chemistry may be complicated by issues of spin state changes, with notable consequences on reaction rates and selectivities.

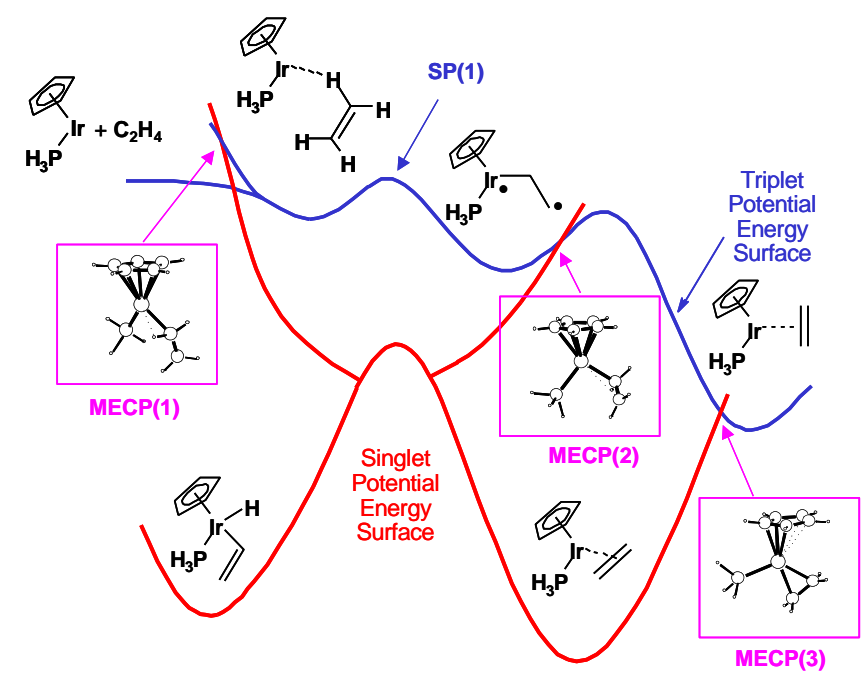

Figure 20. Schematic drawing of the competitive coordinative and oxidative additions of ethylene to $\mathrm{CpIr}\left(\mathrm{PH}_{3}\right)$. Adapted from ref. [68].

There is still much to be learned in this area. In particular, it is necessary to acquire a better understanding of how the coordination sphere (nature of the ligands and the coordination geometry) determines the ground state of an open-shell compound though the independent influence on the electron pairing energy and on the relevant orbital gap. Since coordination geometries may be enforced by the ligands nature (through their rigidity and steric bulk), ligand design is expected to play a determining role in this area. It is necessary to learn more about the basic reactivity of complexes having the same metal and coordination environment but different spin. In this way, enforcement of the desired ground state may lead to the improvement of certain reaction selectivities and catalytic efficiencies. Improved algorithms for a faster computational scanning of spin crossover reactions will also benefit 
this area of research. The computational identification of the details, in a reliable way, for a spin crossover pathway, either alone or in competition with other pathways, is crucial for the understanding and the further improvement of a chemical process. For stoichiometric or catalytic processes involving spin crossover, it is also necessary to better understand and ultimately control the energy of the MECP with respect to the other relevant stationary points along the reaction coordinate. In summary, a fuller understanding of the structure, stability and reactivity of open shell organometallics and a greater predictive power in this area will strongly impact the future of the stoichiometric and catalytic applications of organometallic compounds.

\section{Acknowledgment}

Through the years, my activity in this area has been supported by a number of funding agencies in the USA and in France. I particularly thank the National Science Foundation (USA) for funding this program during its very first steps through a PYI Award, and later with a regular research grant. I also gratefully acknowledge the Conseil Régional de Bourgogne. Current activities are supported by CINES (grant of free computational time) and by the CNRS.

\section{References}

[1] E. Fischer, W. Hafner, Z. Naturforsch. Sect. B 1954, 9, 503-504.

[2] R. Poli, Chem. Rev. 1996, 96, 2135-2204.

[3] R. H. Crabtree, The Organometallic Chemistry of the Transition Metals, 3rd ed., Wiley-Interscience, New York, 2001.

[4] R. Poli, J. N. Harvey, Chem. Soc. Rev 2003, 32, 1-8.

[5] J. N. Harvey, R. Poli, K. M. Smith, Coord. Chem. Rev. 2003, 238-239, 347-361.

[6] S. Shaik, D. Danovich, A. Fiedler, D. Schröder, H. Schwarz, Helv. Chim. Acta 1995, 78, 1393-1407.

[7] D. Schröder, S. Shaik, H. Schwarz, Acc. Chem. Res. 2000, 33, 139-145.

[8] C. A. Tolman, Chem. Rev. 1977, 77, 313-348.

[9] N. H. Buttrus, C. Eaborn, P. B. Hitchcock, J. D. Smith, A. C. Sullivan, J. Chem. Soc., Chem. Commun. 1985, 1380-1381.

[10] B. K. Bower, H. G. Tennent, J. Am. Chem. Soc. 1972, 94, 2512-2514. 
[11] Only the $\sigma$ electrons that a single donor element furnishes are usually counted for the EAN.

[12] M. Mediati, G. N. Tachibana, C. M. Jensen, Inorg. Chem. 1992, 31, 1827-1832.

[13] K. G. Caulton, New J. Chem. 1994, 18, 25-41.

[14] A. A. Gonzalez, S. L. Mukerjee, S.-J. Chou, Z. Kai, C. D. Hoff, J. Am. Chem. Soc. 1988, 110, 4419-4421.

[15] B. Hessen, J. H. Teuben, T. H. Lemmen, J. C. Huffman, K. G. Caulton, Organometallics 1985, 4, 946-948.

[16] E. O. Fischer, K. Ulm, P. Kuzel, Z. Anorg. Allg. Chem. 1963, 319, 253-265.

[17] W. Seidel, K. Fischer, K. Schmiedeknecht, Z. Anorg. Allg. Chem. 1972, 390, 273-279.

[18] S. Hao, S. Gambarotta, C. Bensimon, J. Am. Chem. Soc. 1992, 114, 3556-3557.

[19] R. A. Andersen, E. Carmona-Guzman, J. F. Gibson, G. Wilkinson, J. Chem. Soc., Dalton Trans. 1976, 2204-2211.

[20] R. J. Morris, G. S. Girolami, Organometallics 1989, 8, 1478-1485.

[21] F. Calderazzo, G. Fachinetti, C. Floriani, J. Am. Chem. Soc. 1974, 96, 3695-3696.

[22] K. L. T. Wong, H. H. Brintzinger, J. Am. Chem. Soc. 1975, 97, 5143-5146.

[23] E. Collange, D. Duret, R. Poli, J. Chem. Soc., Dalton Trans. 1999, 875-880.

[24] C. Q. Simpson, Ii, M. B. Hall, M. F. Guest, J. Am. Chem. Soc. 1991, 113, 2898-2903.

[25] I. Cacelli, D. W. Keogh, R. Poli, A. Rizzo, J. Phys. Chem. A 1997, 101, 9801-9812.

[26] M. L. H. Green, P. Mountford, Chem. Soc. Rev. 1992, 29-38.

[27] O. M. Heigl, E. Herdtweck, S. Grasser, F. H. Köhler, W. Strauss, H. Zeh, Organometallics 2002, 21, 3572-3579.

[28] W. Hübel, R. Merényi, J. Organomet. Chem. 1964, 2, 213-221.

[29] B. S. Buyuktas, M. M. Olmstead, P. P. Power, Chem. Commun. 1998, 1689-1690.

[30] W.-Y. Yeh, S.-M. Peng, G.-H. Lee, J. Organomet. Chem. 1999, 572, 125-129.

[31] F. Abugideiri, J. C. Fettinger, D. W. Keogh, R. Poli, Organometallics 1996, 15, 44074416.

[32] I. Cacelli, R. Poli, E. A. Quadrelli, A. Rizzo, K. M. Smith, Inorg. Chem. 2000, 39, 517-524.

[33] P. Legzdins, W. S. Mcneil, S. J. Rettig, K. M. Smith, J. Am. Chem. Soc. 1997, 119, 3513-3522.

[34] P. Legzdins, W. S. Mcneil, K. M. Smith, R. Poli, Organometallics 1998, 17, 615-622.

[35] D. G. Musaev, K. Morokuma, J. Phys. Chem. 1996, 100, 11600-11609.

[36] R. J. Ryther, E. Weitz, J. Phys. Chem. 1991, 95, 9841-9852.

[37] W. Wang, A. A. Narducci, P. G. House, E. Weitz, J. Am. Chem. Soc. 1996, 118, 8654-8657.

[38] W. Wang, E. Weitz, J. Phys. Chem. A 1997, 101, 2358-2363.

[39] A. Veige, L. Slaughter, E. Lobkovsky, P. Wolczanski, N. Matsunaga, S. Decker, T. Cundari, Inorg. Chem. 2003, 42, 6204-6224.

[40] C. Costentin, M. Robert, J. Saveant, J. Am. Chem. Soc. 2003, 125, 105-112.

[41] N. Koga, K. Morokuma, Chem. Phys. Lett. 1985, 119, 371-374.

[42] A. Farazdel, M. Dupuis, J. Comput. Chem. 1991, 12, 276-282.

[43] D. R. Yarkony, J. Phys. Chem. 1993, 97, 4407-4412.

[44] M. J. Bearpark, M. A. Robb, H. B. Schlegel, Chem. Phys. Lett. 1994, 223, 269-274.

[45] J. N. Harvey, M. Aschi, H. Schwarz, W. Koch, Theor. Chem. Acc. 1998, 99, 95-99.

[46] K. M. Smith, R. Poli, J. N. Harvey, New J. Chem. 2000, 24, 77-80.

[47] J. S. Hess, S. Leelasubcharoen, A. L. Rheingold, D. J. Doren, K. H. Theopold, J. Am. Chem. Soc. 2002, 124, 2454-2455.

[48] D. W. Keogh, R. Poli, J. Am. Chem. Soc. 1997, 119, 2516-2523.

[49] M. W. George, M. T. Haward, P. A. Hamley, C. Hughes, F. P. A. Johnson, V. K. Popov, M. Poliakoff, J. Am. Chem. Soc. 1993, 115, 2286-2299. 
[50] V. R. Jensen, R. Poli, J. Phys. Chem. A 2003, 107.

[51] P. L. Bogdan, E. Weitz, J. Am. Chem. Soc. 1989, 111, 3163-3167.

[52] P. L. Bogdan, E. Weitz, J. Am. Chem. Soc. 1990, 112, 639-644.

[53] J. L. Detrich, O. M. Reinaud, A. L. Rheingold, K. H. Theopold, J. Am. Chem. Soc. 1995, 117, 11745-11748.

[54] J.-L. Carreón-Macedo, J. N. Harvey, J. Am. Chem Soc. in press.

[55] C. Hall, W. D. Jones, R. J. Mawby, R. Osman, R. N. Perutz, M. K. Whittlesey, J. Am. Chem. Soc. 1992, 114, 7425-7435.

[56] M. K. Whittlesey, R. J. Mawby, R. Osman, R. N. Perutz, L. D. Field, M. P. Wilkinson, M. W. George, J. Am. Chem. Soc. 1993, 115, 8627-8637.

[57] S. A. Macgregor, O. Eisenstein, M. K. Whittlesey, R. N. Perutz, J. Chem. Soc., Dalton Trans. 1998, 291-300.

[58] J. C. Green, J. N. Harvey, R. Poli, J. Chem. Soc, Dalton Trans. 2002, 1861-1866.

[59] A. A. Cole, J. C. Fettinger, D. W. Keogh, R. Poli, Inorg. Chim. Acta 1995, 240, 355366.

[60] A. H. Janowicz, H. E. Bryndza, R. G. Bergman, J. Am. Chem. Soc. 1981, 103, 15161518.

[61] R. Poli, K. M. Smith, Eur. J. Inorg. Chem. 1999, 877-880.

[62] K. M. Smith, R. Poli, P. Legzdins, Chem. Commun. 1998, 1903-1904.

[63] K. M. Smith, R. Poli, P. Legzdins, Chem. Eur. J. 1999, 5, 1598-1608.

[64] P. Hofmann, Angew. Chem., Int. Ed. Engl. 1977, 16, 536-537.

[65] We shall not address the more trivial situation of side product formation from consecutive reactions.

[66] P. O. Stoutland, R. G. Bergman, J. Am. Chem. Soc. 1988, 110, 5732-5744.

[67] J. Silvestre, M. J. Calhorda, R. Hoffmann, P. O. Stoutland, R. G. Bergman, Organometallics 1986, 5, 1841-1851.

[68] K. M. Smith, R. Poli, J. N. Harvey, Chem. Eur. J. 2001, 7, 1679-1690. 


\section{Graphical abstract}

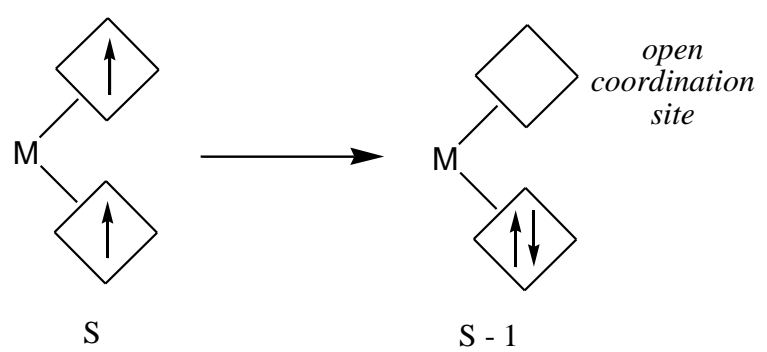

\section{Synopsis}

The rationalization of the stability, physical properties, and chemical reactivity of openshell organometallic compounds has witnessed tremendous progress in recent years. Effects associated to spin state changes are particularly relevant for reaction rates and selectivities. The synergy of experimentations and high-level computations have resulted in an unprecedented level of understanding. 